\title{
Pubertal Adolescent Male-Female Differences in Insulin Sensitivity and Glucose Effectiveness Determined by the One Compartment Minimal Model
}

\author{
ROBERT P. HOFFMAN, PAOLO VICINI, WILLIAM I. SIVITZ, AND CLAUDIO COBELLI \\ Departments of Pediatrics [R.P.H.] and Internal Medicine [W.I.S.], University of Iowa College of \\ Medicine, Iowa City, Iowa 52242, U.S.A.; the Department of Bioengineering [P.V.], University of \\ Washington, Seattle, Washington 98195, U.S.A.; and the Department of Electronics and Informatics \\ [C.C.], University of Padua, Padua, Italy 35131
}

\begin{abstract}
ABST
Most studies of insulin sensitivity in puberty have been
cross-sectional and have not been able to longitudinally address
changes that might occur. In addition, these studies were unable
to separate out glucose's ability to stimulate its own disposal
(glucose effectiveness, $\mathrm{S}_{\mathrm{G}}$ ) from insulin sensitivity ( $\mathrm{S}_{\mathrm{I}}$ ) or to
separate the hepatic and peripheral effects of insulin. To address
these problems, we used the frequently sampled i.v. glucose
tolerance test with [6,6] $\mathrm{D}_{2}$ glucose to study $\mathrm{S}_{\mathrm{G}}^{*}$ and $\mathrm{S}_{\mathrm{I}}^{*}$ in 24
children (Tanner stage $1-3$ ) at 6-mo intervals over an 18-mo
period. Mean overnight GH and fasting GH binding protein
(GHBP), IGF-1, and leptin levels were also measured. $\mathrm{S}_{\mathrm{G}}^{*}$ did not
differ between the sexes or Tanner stages. $\mathrm{S}_{\mathrm{I}}^{*}$ did not differ
between Tanner stages for either sex and was higher in boys than
in girls. Hepatic insulin resistance did not differ between sexes or
Tanner stages. $\mathrm{S}_{\mathrm{G}}^{*}$ was not related to any of the other variables
measured. $\mathrm{S}_{\mathrm{I}}^{*}$ was negatively related to BMI, GHBP, IGF1, and
\end{abstract}
leptin. These results demonstrate that insulin sensitivity is greater in prepubertal and early pubertal boys than in girls and is primarily determined by body mass effects.(Pediatr Res 48: 384-388, 2000)
BMI, body mass index

\section{Abbreviations}
GHBP, GH binding protein
IGF1, insulin-like growth factor 1
$\mathbf{S}_{\mathbf{G}}$, glucose effectiveness
$\mathbf{S}_{\mathbf{G}}^{*}$, glucose effectiveness determined from labeled glucose and one compartment minimal model
$\mathbf{S}_{\mathbf{I}}$, insulin sensitivity
$\mathbf{S}_{\mathbf{I}}^{*}$, insulin sensitivity determined from labeled glucose and one compartment minimal model

Multiple cross-sectional studies have demonstrated that insulin sensitivity decreases as children enter puberty (1-7). These studies have been done with either hyperinsulinemic or hyperglycemic glucose clamp $(1-3,5)$ or with the tolbutamide modified frequently sampled i.v. glucose tolerance test and the one-compartment minimal model $(4,6,7)$. The decrease in insulin sensitivity as children progress through puberty has generally been attributed to increasing body mass and $\mathrm{GH}$ effect as evidenced by negative relationships between insulin sensitivity and body mass index (BMI) $(8,9)$ and IGF-1 $(4,6$, 7 ). These studies do not allow one to follow the course of changes in insulin sensitivity as children progress through

Received December 8, 1999; accepted March 9, 2000.

Correspondence: Robert P. Hoffman, M.D., Children's Hospital of Iowa, 200 Hawkins Drive, Iowa City, Iowa, 52242, U.S.A.

This study was funded by a grant from the Genentech Foundation for Growth and Development and by the General Clinical Research Center of the University of Iowa (RR59). puberty or to determine the time course of specific changes as children progress through puberty.

A second concern is which method of assessing insulin sensitivity is more appropriate. The glucose clamp technique cannot distinguish between glucose's ability to stimulate its own uptake (glucose effectiveness) and insulin's effect on glucose uptake, which is an advantage of the frequently sampled i.v. glucose tolerance test (10). Secondly most studies have not used labeled glucose to separate the peripheral and hepatic effects of insulin.

To correct these problems we used the stable-labeled frequently sampled i.v. glucose tolerance test and one compartment minimal model $(9,11)$ to assess changes in insulin sensitivity at 6 -mo intervals in 24 children between 8 and 13 y of age over an 18-mo period. In addition, to further assess the relationships between GH action, obesity, and insulin sensitivity, we also measured overnight GH secretion and fasting $\mathrm{GH}$ binding protein (GHBP), IGF-1, and leptin levels. GHBP was 
measured because it is a marker of peripheral GH receptor number (12) and IGF-1 because it is an indicator of GH action. Leptin was measured because it is closely related to body mass index and total body fat (13).

\section{METHODS}

Subjects. Twenty-four children (age: $10.7 \pm 1.6 \mathrm{y}$, mean \pm $\mathrm{SD} ; 11$ girls) were enrolled in the study. Mean BMI at enrollment was $18.4 \pm 3.6 \mathrm{~kg} \mathrm{~m}^{-2}$. All were healthy and were on no medications. Informed consent was obtained from the legal guardian and informed assent from the participant. The University of Iowa Institutional Review Board for Human Investigation approved the protocol.

Measures. Plasma glucose concentration was measured using a YSI Model 2300 STAT Glucose Analyzer (Yellow Springs Instruments, Yellow Springs, OH, U.S.A.). Plasma insulin and GH were measured by RIA in the CORE laboratory of the Clinical Research Center of the University of Iowa. Leptin was measured by RIA from a commercial kit (Linco, Inc, St. Louis, MO, U.S.A.) with coefficients of variation from $3 \%$ to $11 \%$. Plasma samples were sent to Endocrine Sciences (Callabassas Hills, CA, U.S.A.) for measurement of IGF-1 and GHBP concentrations.

Mole percent excess of $[6,6] \mathrm{D}_{2}$ glucose was measured in the Mass Spectrometry Laboratory of the University of Iowa using an HP series Mass Selective Detector equipped with a HP 5890 series gas chromatography and a HP 7673 autosampler. The calibration curve was used for determining the ratio of $\mathrm{D}_{2}$ to $\mathrm{D}_{2}$ $+\mathrm{D}_{0}\left(\mathrm{D}_{0}\right.$ nondeutyrated glucose $)$ and was linear over the range of 0.87 to $20.84 \%$.

Protocol. Each subject was admitted to the Clinical Research Center (CRC) at The University of Iowa at $2000 \mathrm{~h}$. Subjects were instructed by the CRC dietician to consume a high carbohydrate diet for $72 \mathrm{~h}$ before study admission. An i.v. catheter was placed in the antecubital fossa of one arm. At 2200 the lights were extinguished and the subject asked to try to sleep. Blood samples were then drawn every $20 \mathrm{~min}$ through 0800 the next morning for measurement of plasma GH concentration (6). At 0800 blood was also drawn for measurement of plasma leptin, GHBP, and IGF-1 concentrations. A second i.v. catheter was inserted then for infusion of $25 \%$ dextrose in water with $13.3 \pm 2.7 \%[6,6] \mathrm{D}_{2}$ glucose (9). Three milliliter blood samples were taken at $-10,0,2,4,6,8,12,14,16,19$, $22,27,32,42,52,62,72,82,92,102,122,142,162$, and 182 min relative to a glucose bolus for measurement of plasma glucose, insulin, and $[6,6] \mathrm{D}_{2}$ glucose concentrations.

An experienced pediatric endocrinologist performed Tanner staging of breasts or genitalia in females and males, respectively.

Calculations. Insulin sensitivity $\left(\mathrm{S}_{\mathrm{I}}^{*}\right)$ and glucose effectiveness $\left(\mathrm{S}_{\mathrm{G}}^{*}\right)$ were calculated using the one compartment minimal model for labeled glucose $(9,11)$. Traditional $\mathrm{S}_{\mathrm{I}}^{*}$ and $\mathrm{S}_{\mathrm{G}}^{*}$ values from the one compartment model were multiplied by the volume of distribution to give clearance units.

The incremental insulin response to glucose was calculated as the area under the insulin curve from 0 to 60 min using the trapezoidal rule. Hepatic glucose production was calculated over the last hour of the study when total glucose concentrations had returned to a quasi steady state through the use of Steele's equations (14). To adjust for differing insulin levels between subjects during the time period when hepatic glucose production was determined, hepatic insulin resistance was calculated by multiplying hepatic glucose production by the mean insulin level over the same hour since higher insulin levels should suppress hepatic glucose production (9).

Statistics. Analysis of variance was used to detect differences between sexes and Tanner stage. Since most of the variables studied were significantly related to BMI, it was included as a covariate in the appropriate analysis. If a child was the same Tanner stage at two or more visits, only the data from the last visit at the given Tanner stage was used. Planned contrasts were used for individual comparisons. Data were log normalized when necessary. Linear regression analysis was used to assess relationships between variables. Results are expressed as mean $\pm \mathrm{SE}$.

\section{RESULTS}

Plasma glucose and insulin levels (Figs. 1 and 2). Plasma glucose levels did not differ between sexes or Tanner stages. Fasting plasma insulin levels during the test did vary between sexes $\left(F_{1-41}=4.88, p=0.033\right)$ in all subjects; however, this difference was not significant in any of the individual Tanner stages. Fasting insulin did not differ between Tanner stages in either sex. BMI was a significant covariate $\left(F_{1-41}=7.24, p=\right.$ $0.01)$. The incremental insulin response to glucose loading differed between sexes $\left(F_{1-38}=23.5, p<0.001\right)$ but no effect of Tanner stage was found. BMI was again found to a be significant covariate $\left(F_{1-38}=55.5, p<0.001\right)$. The sex difference for incremental insulin response was significant in Tanner stages $1(p=0.050)$ and $3(p<0.001)$ and tended to be different in Tanner stage $4(p=0.092)$. The sex by Tanner

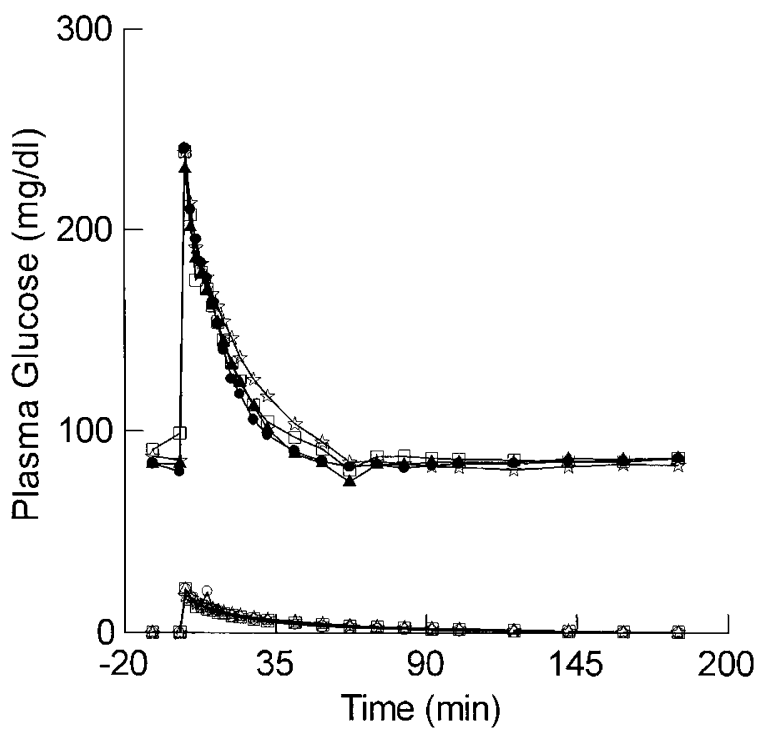

Figure 1. Mean plasma total (upper) and labeled (lower) glucose concentrations in the different Tanner stages for both sexes. Tanner stage 1: closed circles; Tanner stage 2: open triangles; Tanner stage 3: closed squares; Tanner stage 4: open stars. 

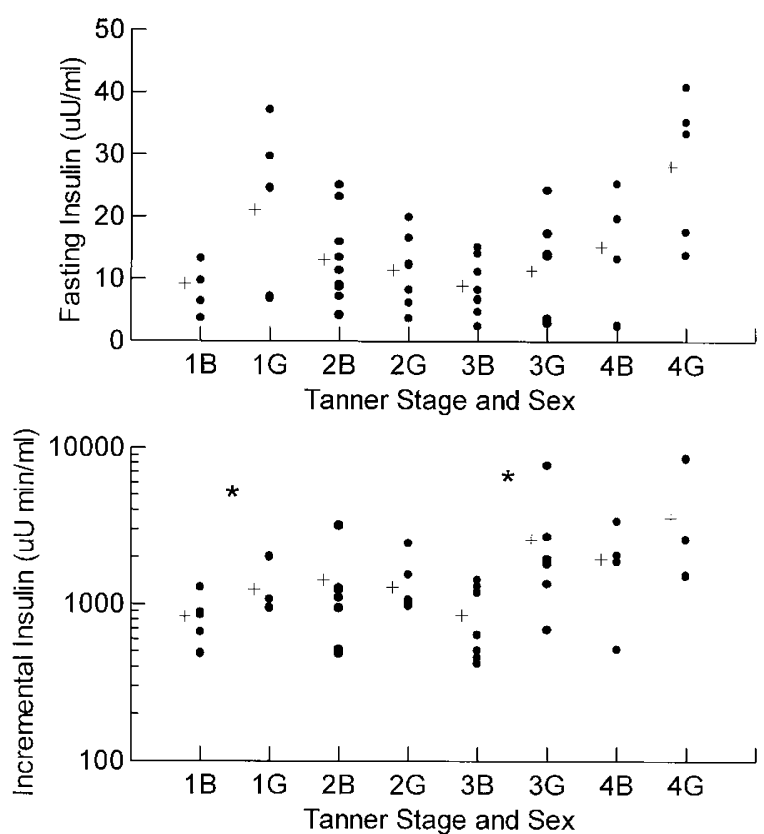

Figure 2. Fasting plasma insulin and incremental insulin response to glucose loading over the 1 st hour of the study according to Tanner stage (number) and sex (B, boys; $\mathrm{G}$, girls). + Mean value. ${ }^{*} p<0.05$ between sexes when adjusted for BMI.

stage interaction was not significant $\left(F_{3-41}=2.273, p=\right.$ 0.094).

Glucose effectiveness and peripheral and hepatic insulin sensitivity (Figs. 3 and 4). Analysis of variance with Tanner stage and sex as grouping factors and BMI as a covariate revealed that $\mathrm{S}_{\mathrm{I}}^{*}$, but not $\mathrm{S}_{\mathrm{G}}^{*}$, differed between the sexes $\left(F_{1-37}\right.$ $=5.7, p<0.001)$ in the group as a whole. No Tanner stage or

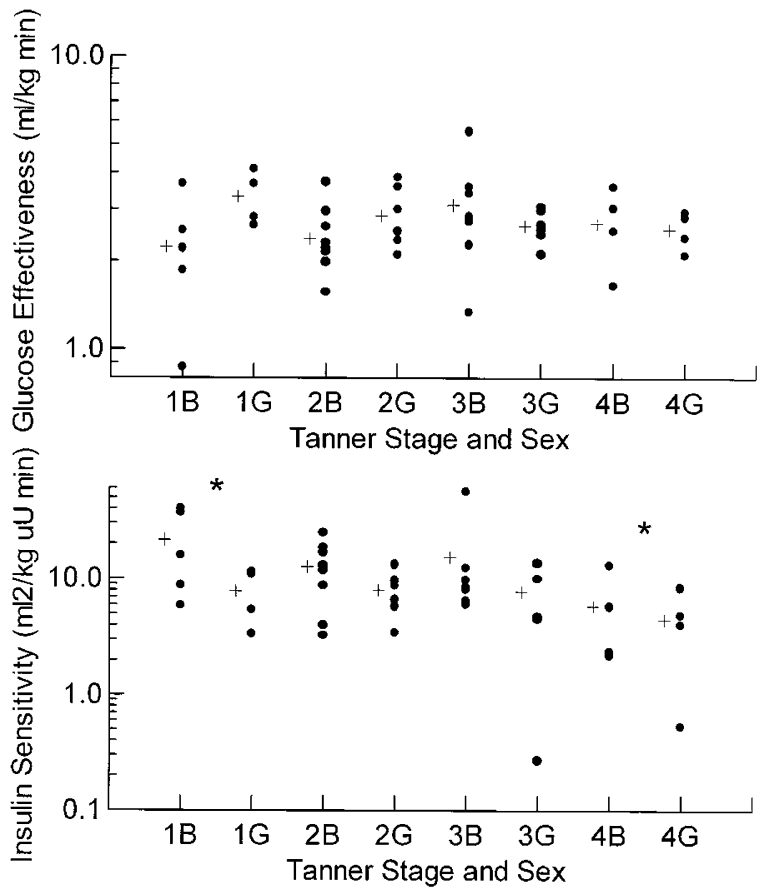

Figure 3. Glucose effectiveness and insulin sensitivity according to Tanner stage (number) and sex (B, boys; $\mathrm{G}$, girls). + Mean value. ${ }^{*} p<0.05$ between sexes when adjusted for BMI.

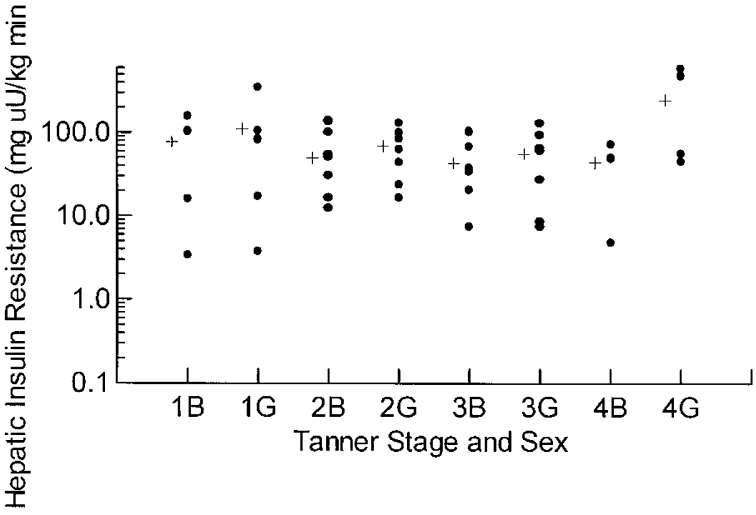

Figure 4. Hepatic insulin resistance according to Tanner stage (number) and sex (B, boys; $\mathrm{G}$, girls). + Mean value. ${ }^{*} p<0.05$ between sexes when adjusted for BMI.

sex by Tanner stage effects were present. BMI was a significant covariate for $\mathrm{S}_{\mathrm{I}}^{*}\left(F_{1-37}=15.6, p<0.001\right)$. For the individual Tanner stages the difference in $\mathrm{S}_{\mathrm{I}}^{*}$ between males and females was significant in Tanner stages $1(p=0.016)$ and $4(p=$ $0.044)$ and tended to be significant in Tanner stages $2(p=$ $0.082)$ and $3(p=0.061)$. Hepatic glucose production and insulin resistance did not differ between the sexes or with BMI.

Relationship to other hormones (Tables 1 and 2). Mean levels for BMI, leptin, overnight GH, GHBP, and IGF-1 are shown in Table 1. BMI did not differ between the sexes or Tanner stages. For the other variables, analysis of variance with BMI as a covariant revealed significant differences between Tanner stages for mean overnight GH $\left(F_{3-42}=5.99\right.$, $p=0.0017)$ and IGF-1 $\left(F_{3-42}=11.6, p<0.001\right)$. There tended to be differences between sexes for leptin $\left(F_{1-38}=2.9\right.$, $p=0.095)$. No significant sex by Tanner stage interactions for any of the variables were found.

Because of the sex differences in $\mathrm{S}_{\mathrm{G}}^{*}$ and $\mathrm{S}_{\mathrm{I}}^{*}$, the correlations with other hormones were studied separately in each sex. In girls $\mathrm{S}_{\mathrm{G}}^{*}$ decreased as IGF-1 increased. This relationship was not found in boys. Stepwise linear regression in girls initially

Table 1. Mean body mass index (BMI) and leptin, overnight GH, $G H$ binding protein (GHBP), and IGF-1 levels in children as they progress through puberty

\begin{tabular}{lcccc}
\hline & \multicolumn{4}{c}{ Tanner stage } \\
\cline { 2 - 5 } & 1 & 2 & 3 & 4 \\
\hline BMI $\left(\mathrm{kg} / \mathrm{m}^{2}\right)$ & & & & \\
Boys & $18.1 \pm 1.7$ & $19.5 \pm 1.7$ & $18.6 \pm 1.4$ & $22.5 \pm 3.0$ \\
$\quad$ Girls & $17.2 \pm 1.0$ & $17.3 \pm 0.8$ & $19.9 \pm 1.7$ & $21.1 \pm 2.8$ \\
Leptin (ng/ml) & & & & \\
$\quad$ Boys & $6.1 \pm 2.2$ & $7.1 \pm 2.9$ & $5.2 \pm 1.6$ & $11.6 \pm 6.6$ \\
Girls & $2.9 \pm 0.9$ & $8.5 \pm 2.1$ & $11.0 \pm 2.4$ & $19.1 \pm 13.2$ \\
GH (ng/ml)* & & & & \\
Boys & $2.8 \pm 0.6$ & $3.6 \pm 0.7$ & $3.3 \pm 0.6$ & $7.0 \pm 2.5$ \\
Girls & $2.8 \pm 0.9$ & $3.8 \pm 0.6$ & $4.8 \pm 0.6$ & $5.5 \pm 1.2$ \\
GHBP (pmol/L) & & & & \\
Boys & $963 \pm 110$ & $1060 \pm 120$ & $887 \pm 156$ & $1050 \pm 280$ \\
Girls & $941 \pm 200$ & $979 \pm 74$ & $1150 \pm 150$ & $951 \pm 179$ \\
IGF-1 (ng/ml)* & & & & \\
Boys & $198 \pm 37$ & $231 \pm 16$ & $292 \pm 1.5$ & $396 \pm 17$ \\
Girls & $193 \pm 20$ & $265 \pm 33$ & $380 \pm 38$ & $436 \pm 48$ \\
\hline
\end{tabular}

$* p<0.05$ Tanner stage effect. 
Table 2. Relationships between glucose effectiveness $\left(S_{G}^{*}\right)$ and insulin sensitivity $\left(S_{I}^{*}\right)$ and $G H$ binding protein $(G H B P)$, IGF-1, mean overnight $G H(M G H)$, and leptin as children progress through puberty for boys and girls.

\begin{tabular}{|c|c|c|c|c|c|c|c|c|}
\hline & \multicolumn{4}{|c|}{$\mathrm{S}_{\mathrm{G}}^{*}$} & \multicolumn{4}{|c|}{$\mathrm{S}_{\mathrm{I}}^{*}$} \\
\hline & \multicolumn{2}{|c|}{ Boys } & \multicolumn{2}{|c|}{ Girls } & \multicolumn{2}{|c|}{ Boys } & \multicolumn{2}{|c|}{ Girls } \\
\hline & $r$ & $p$ & $r$ & $p$ & $r$ & $p$ & $r$ & $p$ \\
\hline GHBP & -0.31 & 0.13 & -0.33 & 0.14 & -0.49 & 0.012 & -0.73 & $<0.001$ \\
\hline IGF-1 & 0.28 & 0.17 & -0.63 & 0.003 & -0.47 & 0.017 & -0.44 & 0.041 \\
\hline MGH & -0.28 & 0.17 & 0.35 & 0.12 & -0.17 & 0.41 & 0.35 & 0.12 \\
\hline Leptin & -0.16 & 0.41 & -0.38 & 0.086 & -0.46 & 0.022 & -0.45 & 0.041 \\
\hline
\end{tabular}

including these variables and BMI found that $38 \%(p=0.003)$ of the variability in $\mathrm{S}_{\mathrm{G}}^{*}$ could be explained by the following equation: $\ln \left(\mathrm{S}_{\mathrm{G}}^{*}\right)=+1.348-0.001 \times \mathrm{IGF} 1$.

$\mathrm{S}_{\mathrm{I}}^{*}$ in both sexes was negatively correlated with IGF-1, GHBP, IGF-1, and leptin. Stepwise linear regression including all significant variables in girls revealed that the following equation predicted $69 \%(p<0.001)$ of the variance in $\mathrm{S}_{\mathrm{I}}^{*}, \ln \mathrm{S}_{\mathrm{I}}^{*}$ $=13.6-4.1 \times \ln (\mathrm{BMI})$. In boys also, only the relationship with BMI was included in the equation, $\ln \mathrm{S}_{\mathrm{I}}^{*}=9.7-2.5 \times \ln$ (BMI), which predicted $48 \%$ of the variance.

Relationship to height and weight velocity. In boys only, height velocity was positively related to IGF-1 levels $(r=$ $0.45, p=0.038)$. Height velocity was not significantly related to either $\mathrm{S}_{\mathrm{G}}^{*}$ or $\mathrm{S}_{\mathrm{I}}^{*}$ in either sex. In girls weight velocity was positively related to BMI $(r=0.55, p=0.019)$, GHBP $(r=$ $0.61, p=0.007)$, IGF1 $(r=0.55, p=0.017)$, and incremental insulin response $(r=0.57, p=0.012)$. If BMI was included in the regression equation the last three relationships were no longer significant. Weight velocity was not related to $\mathrm{S}_{\mathrm{G}}^{*}$ or $\mathrm{S}_{\mathrm{I}}^{*}$ in either sex.

\section{DISCUSSION}

These results demonstrate that girls are less insulin sensitive than boys are, but compensate for the decreased sensitivity by increasing their insulin secretion. Changes in $\mathrm{S}_{\mathrm{I}}^{*}$ during progression through puberty are not present when changes in body mass are accounted for.

Most studies of pubertal changes in insulin sensitivity do not mention the presence or absence of sex differences. Cook et al. (6) in a cross-sectional study using the modified frequently sampled glucose tolerance test without labeled glucose and the minimal model did not find differences between sexes for either $\mathrm{S}_{\mathrm{I}}$ or $\mathrm{S}_{\mathrm{G}}$. Travers et al. (7) using the same model found lower $\mathrm{S}_{\mathrm{I}}$ in Tanner stage 3 girls than in Tanner stage 3 boys but found no difference between Tanner stage 2 boys and girls. Arslanian et al. (15) did find lower insulin sensitivity in adolescent girls with type 1 diabetes compared with type 1 diabetic boys. They felt the difference was due to increased GH secretion in girls.

This is the first study to demonstrate consistent sex differences in insulin sensitivity between sexes in healthy adolescents. Girls as a group compensated for the lower insulin sensitivity with higher insulin responses so that the plasma glucose concentrations did not differ between the sexes. No reason for the difference in $S_{I}^{*}$ between the sexes is readily apparent. Unlike Arslanian et al. (15) we found no differences in overnight GH secretion between the two groups nor did we find any evidence of differences in peripheral GH responsiveness, as indicated by GHBP levels, or action, as indicated by IGF1, between the sexes. The differences in insulin sensitivity between the sexes are limited to peripheral insulin actions since we found no differences in hepatic insulin resistance between the sexes.

Our results also differ from other studies in that we found no difference between the various Tanner stages for either sex for insulin sensitivity. This was true even if BMI was not included as a covariate although for $\mathrm{S}_{\mathrm{I}}^{*}$ the effect of Tanner stage approached significance. The lack of significance here may be due to our small sample size with a power of 0.75 to detect a difference at the $p=0.05$ level overall. In addition, many of the studies comparing insulin sensitivity do not separate the subjects into individual Tanner stage groups, which may account for part of the difference. Cook et al. (6) however in their study did divide subjects into Tanner stages and found higher insulin sensitivity in Tanner stage 1 subjects than in Tanner stage 2-5 subjects. Travers et al.(7) found lower insulin sensitivity in Tanner stage 3 than in Tanner stage 2 for girls only. Another possible reason for the difference is that our data are longitudinal as opposed to cross-sectional and the changes within a given individual may be small. On the other hand cross-sectional studies may accentuate BMI differences between groups, which could lead to differences in insulin sensitivity.

Our data, like that of others, confirms that insulin sensitivity in adolescents is determined primarily by body mass and/or peripheral GH action $(2-4,6-9)$. We did find consistent relationships between $\mathrm{S}_{\mathrm{I}}^{*}$ and GHBP, IGF-1, and leptin in both sexes, but these relationships could be accounted for by the relationship between insulin sensitivity and body mass since each of the variables increase as body mass increases. Stepwise multiple linear regression was used to try to determine whether any specific determinants of insulin sensitivity and glucose effectiveness could be found. For $\mathrm{S}_{\mathrm{I}}^{*}$ only the relationship with BMI contributed significantly in both sexes. In addition, if $\mathrm{S}_{\mathrm{I}}^{*}$ is a significant predictor of $\mathrm{GH}$ action, then it should correlate with growth velocity, which we did not find. These findings indicate that body mass is the major predictor of insulin sensitivity in early puberty.

For $\mathrm{S}_{\mathrm{G}}^{*}$ in boys no significant predictors were found while in girls glucose's ability to stimulate its own disposal increased as IGF-1 increased. This relationship persisted even when BMI was included in the equation. Thus changes in peripheral GH 
action may alter glucose effectiveness as children progress through puberty. This cannot be definitely stated since a similar relationship was not found in boys and $\mathrm{S}_{\mathrm{G}}^{*}$ did not vary with Tanner stage while IGF-1 clearly did.

In conclusion, these results demonstrate early pubertal girls are less insulin sensitive than boys and compensate with increased insulin secretion. The reasons for the differences between the sexes are unclear. Since insulin resistance has been linked to development of hypertension and type 2 diabetes, these results emphasize that care should be maintained to minimize the development of obesity in adolescence.

Acknowledgments. The authors thank the nurses of the CRC for their assistance in care for the subjects, Dennis Charkowski, and Yalan Li for measurement of $[6,6] \mathrm{D}_{2}$ glucose and especially Nandhini Subbiah for her measurement of plasma glucose levels, computer data entry, and organizational abilities. We also thank Drs. Eva Tsalikian and Patricia Donohoue who helped by Tanner staging the female subjects.

\section{REFERENCES}

1. Amiel SA, Sherwin RS, Simonson DC, Lauritano AA, Tamborlane WV 1986 Impaired insulin action in puberty: a contributing factor to poor glycemic control in adolescents with diabetes. N Engl J Med 315:215-219

2. Bloch CA, Clemons P, Sperling MA 1987 Puberty decreases insulin sensitivity. J Pediatr 110:481-487
3. Caprio S, Plewe G, Diamond MP, Simonson DC, Boulware S, Sherwin RS, Tamborlane WV 1989 Increased insulin secretion in puberty: a compensatory response to reductions in insulin sensitivity. J Pediatr 114:963-967

4. Cutfield WS, Bergman RN, Menon RK, Sperling MA 1990 The modified minimal model: application to measurement of insulin sensitivity in children. J Clin Endocrinol Metab 70:1644-1650

5. Amiel SA, Caprio, Sherwin RS, Plewe G, Haymond MW, Tamborlane WV 1991 Insulin resistance of puberty: a defect restricted to peripheral glucose metabolism. J Clin Endocrinol Metab 72:277-282

6. Cook JS, Hoffman RP, Stene MA, Hansen JR 1993 Effects of maturational stage on insulin sensitivity during puberty. J Clin Endocrinol Metab 77:725-730

7. Travers SH, Jeffers BW, Bloch CA, Hill JO, Eckel RH 1995 Gender and Tanner stage differences in body composition and insulin sensitivity in early pubertal children. J Clin Endocrinol Metab 80:172-178

8. Arslanian S, Suprasongsin C 1996 Insulin sensitivity, lipids and body composition in childhood: is "Syndrome X" present? J Clin Endocrinol Metab 81:1058-1062

9. Hoffman RP, Armstrong PT 1996 Glucose effectiveness, peripheral and hepatic insulin sensitivity, in obese and lean prepubertal children. Int J Obes 20:521-525

10. Ader M, Pacini G, Yang YJ, Bergman RN 1985 Importance of glucose per se to intravenous glucose tolerance: comparison of the minimal-model prediction with direct measurements. Diabetes 34:1092-1103

11. Avogaro A, Bristow JD, Bier DM, Cobelli C, Toffolo G 1989 Stable-label intravenous glucose tolerance test minimal model. Diabetes 38:1048-1055

12. Martha P Rogol A, Blizzard R, Shaw M, Baumann G 1991 Growth hormone binding protein is inversely related to 24 hour growth hormone release in normal boys. J Clin Endocrinol Metab 73:175-181

13. Ahmed M, Ong KKL, Morell DJ, Cox L, Drayer N, Perry L, Preece MA, Dunger DB 1999 Longitudinal study of leptin concentrations during puberty: sex differences and relationship to changes in body composition. J Clin Endocrinol Metab 84:899-905

14. Steele R 1959 Use of C14-glucose to measure hepatic glucose production following an I.V. glucose load or after injection of insulin. Metabolism 8:512-519

15. Arslanian SA, Heil BV, Becker DJ, Drash AL 1991 Sexual dimorphism in insulin sensitivity in adolescents with insulin dependent diabetes mellitus. J Clin Endocrinol Metab 72:920-926 